\title{
Numerical Investigations of a Vertical Axis Wind Turbine with Variable Pitch
}

\author{
F. Frunzulica ${ }^{1,2}$, C. Olteanu ${ }^{3}$, A. Dumitrache ${ }^{2}$, D. Crunteanu ${ }^{1}$ \\ ${ }^{1}$ Department of Aerospace Engineering \\ POLITEHNICA University of Bucharest \\ Polizu Street no.1-6, RO-011061 Bucharest, Romania
}

Phone/Fax number:+40-722.894.399/+40-21-318.24.33, e-mail: ffrunzi@yahoo.com, crunti_dani2005@yahoo.com

2 "Gh. Mihoc - C. Iacob" Institute of Mathematical Statistics and Applied Mathematics,

Calea 13 Septembrie 13, RO-050711 Bucharest, Romania

Phone/Fax number+40-21-318.24.39/+40-21-318.24.33, e-mail: alexandru.dumitrache@ima.ro

${ }^{3}$ Turbomecanica S.A., Iuliu Maniu 244, sect 6, RO-061126, Bucharest,

e-mail: ciprian.olteanu@yahoo.com

\begin{abstract}
In the last decades, the wind energy became more attractive to Romania, and thus many resources are allocated to develop the energetic systems based on wind energy. In this work the performances of a variable-pitch vertical axis wind turbine were investigated using CFD (ANSYS Fluent). A computational methodology has been developed which uses the moving grid option offered by the ANSYS Fluent code, to evaluate amount of energy extracted from the wind. A transient simulation is done for fixed pitch and variable pitch vertical axis wind turbine using a $k-\omega$ SST (Shear Stress Transport) turbulence model. Main parameters like moment coefficient $\mathrm{C}_{\mathrm{m}}$, and power coefficient $\mathrm{C}_{\mathrm{P}}$ are investigated. The conclusion was that the variable-pitch vertical axis wind turbine has the best power coefficient at low/moderate tip speed ratio.
\end{abstract}

\section{Key words}

VAWT, variable-pitch, CFD, power coefficient.

\section{Theoretical background}

Traditionally NACA 4-digits series have been employed for Darrieus-type VAWTs. The NACA0012 is almost used airfoil for H-type VAWT, but in the last time it shifted to thicker airfoil NACA0015, NACA0018 and NACA0022, for a fast starting [1-4].

The present study focuses on the analysis of a VAWT with 2 blades using NACA0012 airfoil and $\sigma=B c /(2 R)=2 \cdot 0.1 /(2 \cdot 0.3)=0.33$ solidity.

One feature of VAWT (H-type) is that the relative velocities perceived by the blade always change as the blade moves to different azimuthally positions, $\theta$. The figure 1 illustrates typical flow velocities around a rotating VAWT blade at a given azimuthally angle $\theta$, where the velocity $U_{\text {local }}$ represents the induced local velocity ( $a$ is the induction factor - it can be function of azimuthally angle). It should be noted that both the magnitude and the direction of the effective velocity (ideally flow) perceived by the blade, $U_{\text {eff }}$, change in a cyclic manner as the blade rotates through different azimuthally angles. As a result, the aerodynamic loads exerted on the blade, change cyclically with $\theta$.

The variation of the incidence as a function of the azimuthally angle $\varphi$ is given by Eq. 1 .

$$
\begin{aligned}
& \varphi=\theta+\pi / 2, \lambda=T S R, \quad \alpha=\arctan \frac{(1-a) \cos \varphi}{\lambda+(1-a) \sin \varphi}, \\
& \frac{U_{\text {eff }}}{U}=\left\{[\lambda+(1-a) \sin \varphi]^{2}+[(1-a) \cos \varphi]^{2}\right\}^{1 / 2}
\end{aligned}
$$

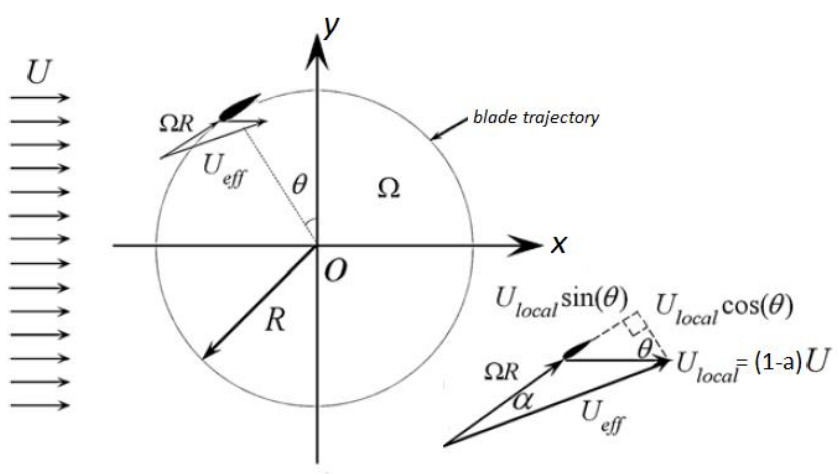

Fig. 1. Velocities around a VAWT

At low TSR, the local angle of attack (theoretical angle) has high values which lead to the dynamic deep stall. Our purpose is to use variable pitch angle to produce positive drive moment, for each blade, at all azimuthal angles. Advantages of the variable pitch are: the turbine can be put into motion without external assistance and at low speed wind and, improved aerodynamic efficiency compared to conventional turbine at low TSR.

The variation law used for the pitch angle has the following formula: 


$$
\begin{aligned}
& \beta_{i}(t)=-\beta_{0} \cos \left(\Omega t+\frac{2 \pi}{N}(i-1)\right), \\
& \beta_{0}=10^{0}, i=1 \ldots N, \Omega=2 \pi n
\end{aligned}
$$

where $\Omega$ is the angular velocity and $\mathrm{N}$ is the number of

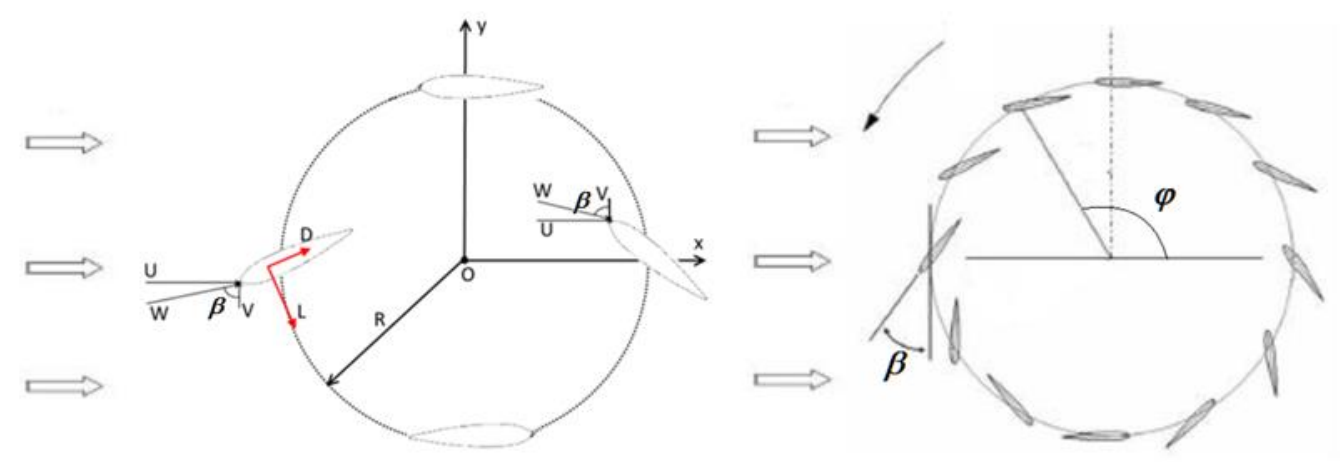

Fig. 2. The variation of the pitch angle on a cycle.

\section{Numerical investigation using CFD. Settings}

The chosen model was 2 blades with NACA0012 airfoil and $\sigma=B c /(2 R)=2 \cdot 0.1 /(2 \cdot 0.3)=0.33$ solidity. Two configurations are investigated: one with fixed blades (M1, fixed pitch angle of $0^{0}$ ) and another one with variable pitch angle of $-10^{0}$ to $+10^{\circ}$ (M2). Numerical investigations were performed for $V_{\infty}=9 \mathrm{~m} / \mathrm{s}$ wind speed. The figure 3 shows the geometry of the computational domain in configuration M1 and M2.

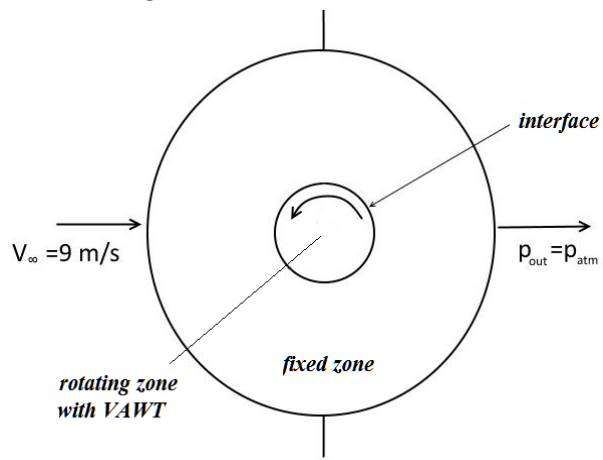

a)

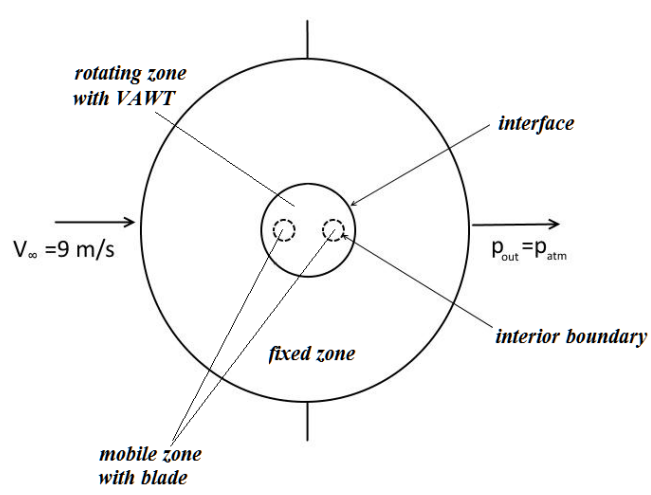

b)

Fig. 3. The geometry of the computational domain:

a) M1; b) M2.
Dynamic mesh strategy. For M1 geometry, the rotating zone (it includes VAWT) has a "rigid body" rotation with constant angular velocity $\Omega$. In the case M2, the motion is more complex and this requires:

- a rotating zone with constant angular velocity $\Omega+$ [deforming zone (springs) + re-meshing]. The re-meshing is performed in the buffer zone which includes 5 layers with triangular elements near interior boundary;

- each blade is surrounded by a circular zone (interior boundary) with rigid motion described by the following formulas (figure 4):

- the position of the mass center (the first blade zone):

$$
x=R \cos (\Omega t), \quad y=R \sin (\Omega t)
$$

- the velocity of the mass center

$$
V_{x}=-R \Omega \sin (\Omega t), V_{y}=R \Omega \cos (\Omega t)
$$

- the angular velocity of the mass center

$$
\Omega_{1}(t)=\Omega+\beta_{0} \Omega \sin (\Omega t)
$$

- for the second blade the same formulas with $180^{\circ}$ phase shift were applied.

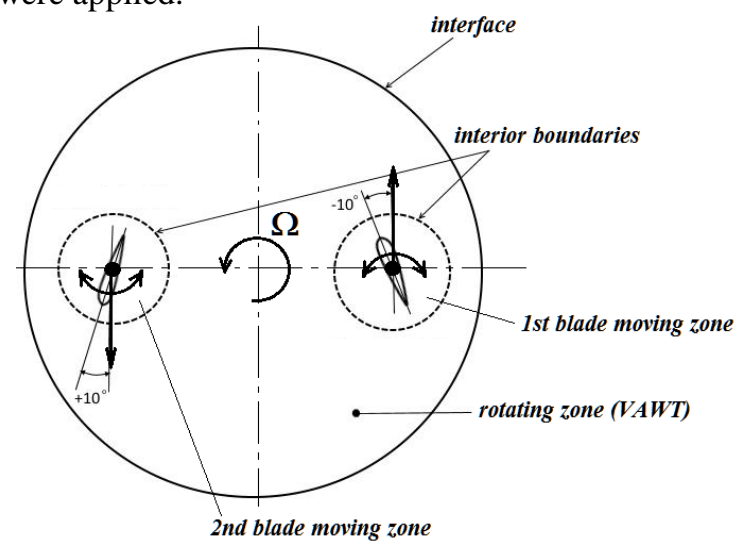

Fig. 4. Dynamic mesh setting for the M2 case

The computational grid has about 400,000 mixed cells (quadrilateral and triangular) and this have been tested for the grid independence of the results. The same 
computational grid was used to start simulations for all cases.

For the present study, unsteady Reynolds averaged Navier-Stokes (URANS) model is the suitable approach to perform simulations with an acceptable computational cost and, at least, reasonable accuracy. For computational simulations the Ansys Fluent code has been used [5].

Due to the incompressibility of the investigated flow, the unsteady pressure-based solver is chosen. All the governing equations for the solution variables, which are decoupled from each other, are solved sequentially and the SIMPLEC algorithm is applied as the pressure-velocity coupling algorithm. With respect to the discretization of the convection terms in the transport equations for the velocity and the turbulence quantities, second-order upwind schemes are utilized. The turbulence model used in the present work was $k-\omega \mathrm{SST}$ (Shear Stress-Transport Turbulence Model).

The numerical time step is based on the characteristic time flow ( $\Delta t=2 \pi / \Omega / 360 \mathrm{~s}$ ); after three revolutions the solution became periodical.

\section{Results}

Figure 5 shown the velocity field in the rotor zone at azimuthal angles between $0^{\circ}$ and $180^{\circ}$, for angular velocity of $\Omega=15 \mathrm{rad} / \mathrm{s}$ ( $2.4 \mathrm{rev} / \mathrm{s})$, corresponding to tip speed ratio of 0.5 , in the left side VAWT with fixed pitch; and in the right side-VAWT with variable pitch [6-9].
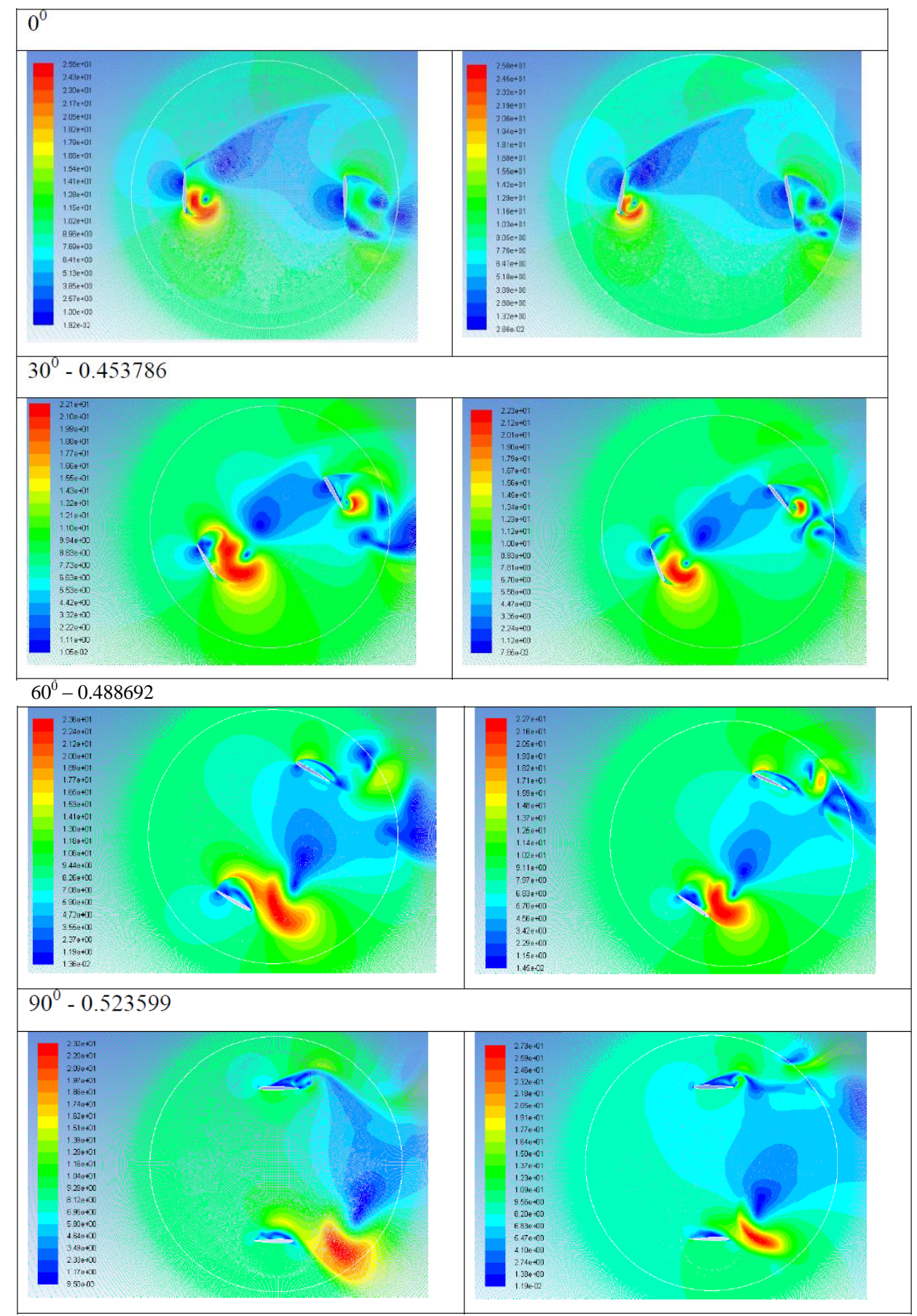

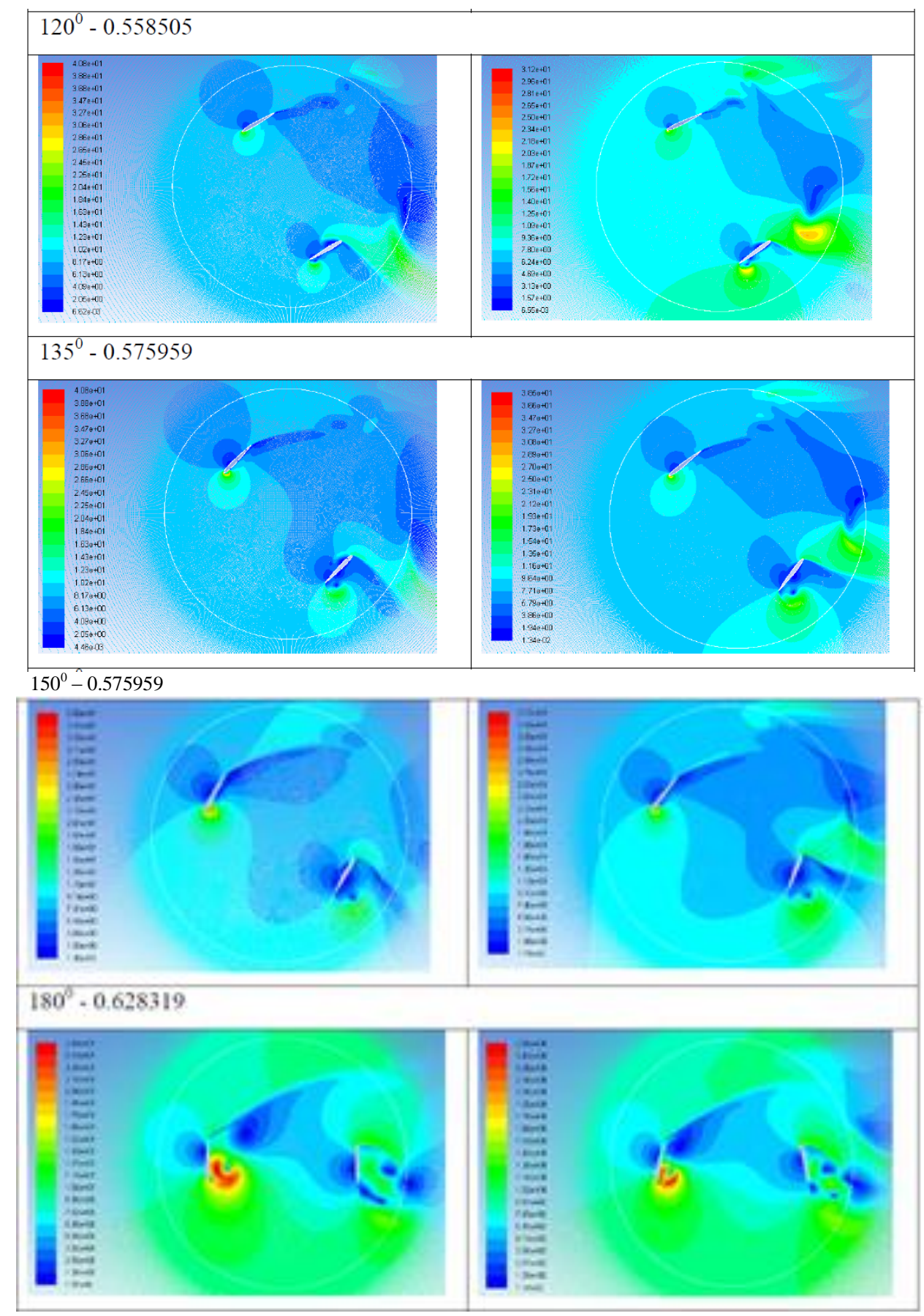

Fig. 5 Velocity field in rotor zone.

The usually coefficients used to estimated performances of the VAWT were defined as follows:

- the moment coefficient relative to the rotation center $(0,0)$ :

$$
C_{m}=\frac{M}{\frac{1}{2} \rho V_{\infty}^{2} c \cdot 1 \cdot c}
$$

- the power coefficient:

$$
C_{P}=\frac{M \Omega}{\frac{1}{2} \rho V_{\infty}^{3} 2 R H}(H=1 \mathrm{~m})
$$

- the average coefficient:

$$
\bar{C}_{P}=\frac{1}{T} \int_{0}^{T} C_{P}(\varphi) d \varphi, T=2 \pi
$$

Figure 6 shown the aerodynamic moment relative to the axis of rotation. From this picture it can be observed that the moment of configuration M2 is greater than configuration M1 (without variable pitch) for low TSR, and for high TSR > 2.5, where the effective angle of attack is low on the blade, the variable pitch has a little effect over performances. 

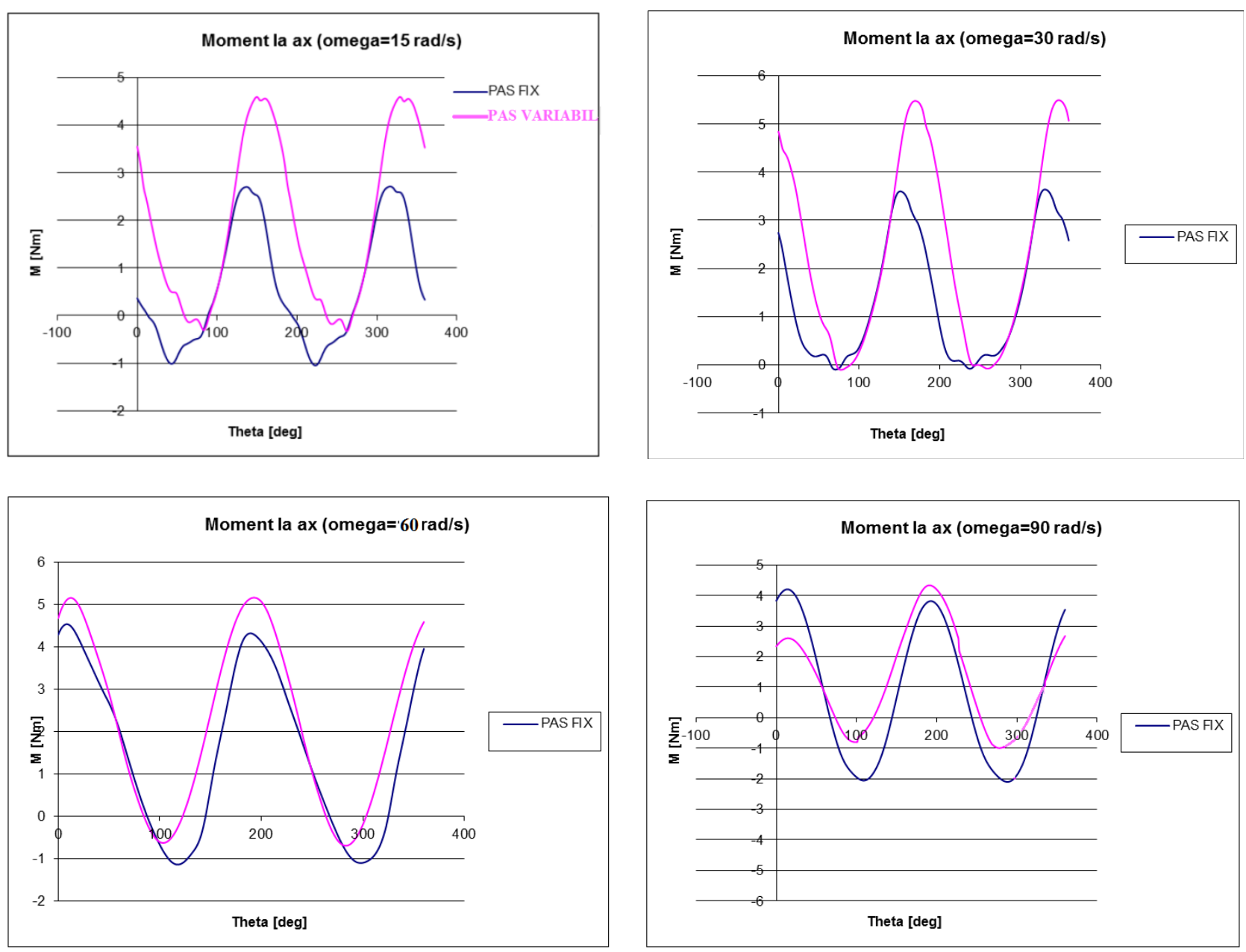

Fig. 6. Aerodynamic moment relative to the axis of rotation

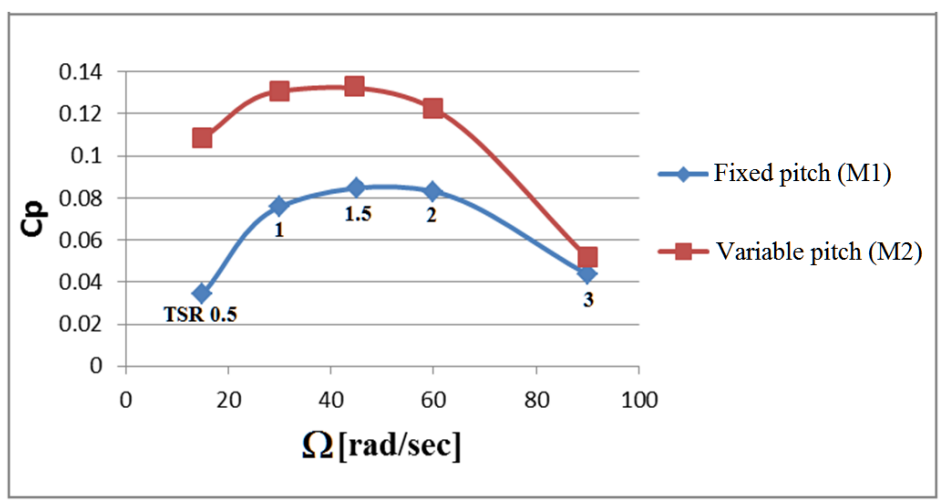

Fig 7. Power coefficient versus angular velocity and TSR.

\section{Conclusion}

It is remarked that the variable pitch lead to augmented power coefficient at low TSR (about 5 times higher) and at TSR greater than 3 the performance of the two turbines tends to be equal. The VAWT requires a control law for pitch angle as function of TSR (to decrease $\beta_{0}$ angle with increasing TSR).

But, technical problems can appears regarding variable pitch, the usually solutions being pitch control with electric actuator (for each blade) or mechanical devices (system with cams).

\section{Acknowledgement}

This work has been achieved through the UPB GEX 2016 Internal Research Grants - Competition 2015- 2016 Contract no. 82/09.26.2016, "System for Renewable Energy Airports" (SERA) 


\section{References}

[1] "HAWT versus VAWT: Small VAWTs find a clear niche" Refocus 4, 2003, pp. 44-46.

[2] I. Nila, R. Bogateanu and M. Stere, "Small power wind turbine (Type Darrieus)", INCAS BULLETIN, 4, 2012, pp. 135-142.

[3] M. Islam, D. S. K. Ting, and A. Fartaj, Aerodynamic Models for Darrieus-Type Straight-Bladed Vertical Axis Wind Turbines, Renewable \& Sustainable Energy Reviews, Vol. 12, 2008, pp. 1087-1109.

[4] L. Lazauskas, Three pitch control system for vertical axis wind turbines compared, Wind Engineering, 16, 1992, pp. 269-282.

[5] *** Ansys Fluent 13. User's guide. Fluent documentation.

[6] I. Paraschivoiu, O. Trifu, and F. Saeed, H-Darrieus wind turbine with blade pitch control, International Journal of Rotating Machinery, 2009, Article ID 505343, 2009, 7 pages; http://dx.doi.org/10.1155/2009/505343.

[7] I. S. Hwang, S. Y. Min, I. O. Jeong, Y. H. Lee and S. J. Kim, Efficiency improvement of a new vertical axis wind turbine by individual active control of blade motion, Proc. SPIE 6173, 617311, 2006; http://dx.doi.org/10.1117/12.658935.

[8] S. J. Chen, Z. Chen, S. Biswas, J. J. Miau, and C. H. Hsieh, Torque and power coefficients of a vertical axis wind turbine with optimal pitch control, ASME 2010-27224, ASME 2010 Power Conference, July 13-15, 2010, Chicago.

[9] N. Fujisawa and M. Takeuchi, Flow Visualization and PIV measurements of Flow Field around a Darrieus Rotor in Dynamic Stall, Journal of Visualization, 1, 1999, pp. 379386. 